\title{
Disfunção do eixo Hipotálamo-Hipófise-Adrenal na dor crônica generalizada: uma análise da literatura com enfoque pericial
}

\author{
Hypothalamus-Pituitary-Adrenal axis dysfunction in chronic \\ widespread pain: literature analysis and disability evaluation
}

Iana Sousa Nascimento ${ }^{1}$, Carmen Silvia Molleis Galego Miziara ${ }^{2}$

\begin{abstract}
Nascimento IS; Miziara CSMG. Disfunção do eixo Hipotálamo-Hipófise-Adrenal na dor crônica generalizada: uma análise da literatura com enfoque pericial. Saúde, Ética \& Justiça. 2015;20(1):29-36.

RESUMO: A dor é sintoma comum e frequente causa de absenteísmo no trabalho e, por ser subjetivo (percepção), é de difícil caracterização pericial. Particularmente na dor crônica musculoesquelética, as faltas recorrentes geram prejuízo econômico substancial para a empresa e para a sociedade. Nesse contexto, o médico perito assume papel importante na avaliação criteriosa de incapacidade laborativa, mas não conta com instrumentos diagnósticos precisos. A possibilidade de existência de marcadores hormonais capazes de elucidar o diagnóstico de dor crônica, sem base anatômica justificável, seria de extremo valor na perícia médica, tornando objetiva a avaliação subjetiva da dor. Com isso em vista, foi feita uma revisão de literatura dos artigos sobre a disfunção do eixo hipotálamo-hipófise-adrenal nessas condições dolorosas publicados nos últimos dez anos, mas alguns artigos mais antigos foram incluídos por suas relevâncias. A literatura médica mostra resultados contraditórios quanto à detecção objetiva de marcadores hormonais em casos de dor crônica sem substrato anatômico. Alguns autores sugerem haver disfunção do eixo hipotálamo-hipófise-adrenal, mas não estabelecem se a relação desta disfunção é com a dor ou com os sintomas psíquicos frequentemente associados. Mais estudos são necessários, com maior número de pacientes estudados.
\end{abstract}

DESCRITORES: Transtornos Somatoformes; Fibromialgia; Dor Crônica; Dor Musculoesquelética; Sistema HipófiseSuprarrenal; Hidrocortisona; Hormônio Adrenocorticotrófico; Hormônio Liberador da Corticotropina.

\footnotetext{
${ }^{1}$ Aluna de pós-graduação em Perícias Médicas da Faculdade de Ciências Médicas da Santa Casa de São Paulo.

${ }^{2}$ Professora assistente da Disciplina de Medicina Legal, Deontologia Médica, Bioética e Perícias Médicas da Faculdade de Medicina do ABC.

Endereço para correspondência: Iana Sousa Nascimento - Rua Oscar Freire, 1758, apt 13A, Pinheiros, São Paulo-SP, 05409011

E-mail: ianapis73@yahoo.com.br
} 


\section{INTRODUÇÃO}

$\mathrm{A}$ dor é um sintoma comum e frequente causa de absenteísmo ${ }^{1}$. Isso se torna um problema nos casos de dor crônica, quando as faltas recorrentes geram um prejuízo econômico substancial para a empresa e para a sociedade. Nesse contexto, o médico perito assume papel importante na avaliação de incapacidade laborativa. A falta de métodos diagnósticos objetivos para a sua conclusão, porém, torna árdua essa tarefa. A possibilidade de utilização de marcadores hormonais que pudessem contribuir de forma prática seria de grande valia, posto que neste tipo de queixa a propedêutica armada é inexistente. $\mathrm{O}$ conhecimento da fisiopatologia da dor, incluindo os mecanismos hormonais envolvidos, assim como de todos os diagnósticos diferenciais se faze necessário neste tipo de perícia, posto que na maioria das vezes a conclusão pericial desta entidade mórbida é embasada em diagnósticos de exclusão.

A dor é dita crônica quando tem duração superior ao tempo esperado de resolução, o que pode corresponder a meses, anos ou toda uma vida ${ }^{2}$. O que se observa muitas vezes na prática médica é que, quanto maior o tempo de duração de uma dor, menor é a chance de ela remitir. Existem diversos tipos de dor crônica de acordo com a sua localização, podendo ser, por exemplo, lombar, cervicobraquial e pélvica. Certamente, uma das mais comuns e desafiadoras é a dor crônica generalizada (DCG). Mais do que limitação para atividades que exijam uso direto da área dolorosa acometida, o indivíduo com dor crônica dita incapacitante habitualmente alega limitação para atividades que têm inclusive impacto mínimo no uso do seu corpo. Como exemplo extremo pode ser citado o uso de sutiã, que, ainda que não configure uma atividade laboral, demonstra o grau intenso da incapacidade alegada. $\mathrm{Na}$ grande maioria das vezes, essa condição clínica está dissociada de uma doença orgânica diagnosticável, isto é, constitui um sintoma sem explicação médica.

A dificuldade em entender esse distúrbio se reflete na dificuldade em nomeá-lo e resulta em diferentes denominações para quadros clínicos semelhantes (fibromialgia, transtorno somatoforme doloroso, síndrome da dor miofascial, síndrome da dor funcional) com pequenas diferenças acadêmicas entre elas. A última edição do Manual Diagnóstico e Estatístico de Transtornos Mentais (DSM-5) ${ }^{3}$ apresentou mudanças na caracterização dos transtornos somatoformes.

De acordo com essa nova versão, os transtornos somatoformes passaram a ser denominados de transtornos de sintomas somáticos (sendo a dor um possível sintoma predominante) e foi frisado o caráter comportamental exagerado em torno dos sintomas, com sofrimento exacerbado autêntico pelo indivíduo em detrimento da ausência de explicação médica para o sintoma somático, sendo que este último aspecto já era reforçado na versão quatro do DSM ${ }^{4}$.

Por sua vez, os critérios diagnósticos de fibromialgia de $2010^{5}$ continuam enfatizando a dor generalizada e significativa, mas valorizam a presença de sintomas somáticos em comparação com os de $1990^{6}$. Apesar das pequenas particularidades, a comunidade científica se pergunta se essas duas doenças não são, na verdade, uma só entidade ${ }^{7-9}$.

Determinar incapacidade laborativa nesses indivíduos é difícil, pois, por um lado, não existem evidências objetivas suficientes para justificar uma incapacidade; por outro lado, a experiência demonstra que, em grande parte das vezes, será praticamente impossível o indivíduo retornar ao trabalho e não raramente ele é aposentado por invalidez $z^{10-11}$.

As tentativas de se comprovar alguma alteração fisiopatológica que justifique não são escassas e se concentram em identificar transtorno encefálico na interpretação da dor ${ }^{12}$. Parte da atenção dos pesquisadores é dirigida para o eixo hipotálamo-hipófise-adrenal (EHHA). Este, junto com o sistema nervoso autônomo (SNA), é responsável por manter a homeostase basal e a resposta ao estresse. O EHHA funciona mediante esquema de feedback, ou retroalimentação, positivo e negativo, por meio de hormônios. Nesse eixo, o hipotálamo secreta o hormônio liberador de corticotrofina (HLC) no sistema portal hipofisário, que estimula a hipófise anterior a secretar hormônio adrenocorticotrófico (ACTH) na circulação sistêmica. O ACTH, por sua vez, estimula o córtex da glândula adrenal a produzir glicocorticóide ${ }^{13-15}$. O cortisol tem vários mecanismos de feedback negativos que inibem a ativação do EHHA e atua sobre o metabolismo dos carboidratos, proteínas e lipídios, assim como modula a resposta anti-inflamatória e imunossupressora, a dor e o ritmo circadiano ${ }^{16-17}$.

Embora o EHHA não esteja envolvido diretamente com o manejo da dor, sabe-se que neurônios produtores de HLC inervam áreas secretoras de propiomelanocortina localizadas no núcleo arqueado do hipotálamo e em áreas que controlam a dor na medula espinal e no rombencéfalo (ponte, cerebelo e bulbo). A ativação do sistema de resposta ao estresse causa secreção de peptídeos derivados da proopiomelanocortina induzidos pelo HLC como também de outros peptídeos opióides, o que aumenta a analgesia ${ }^{14}$.

Sendo um tema controverso tanto no meio assistencial quanto no pericial, a caracterização do quadro de dor crônica como tendo uma possível explicação médica pode ajudar o médico perito a definir melhor a incapacidade laboral desses indivíduos, assim como, eventualmente, levar à utilização de ferramentas objetivas para esse fim.

\section{OBJETIVOS}

Os objetivos desse estudo foram o de descrever a 
existência de alterações hormonais do eixo hipotálamohipófise-adrenal em indivíduos com dor crônica generalizada e discutir se essas alterações hormonais, se existentes, podem ser secundárias ao quadro de dor crônica generalizada ou se podem ser causa da mesma e se poderiam ser utilizadas como um dado adicional, objetivo, pelo médico perito na avaliação de incapacidade desses indivíduos que usualmente apresentam na perícia apenas o fator subjetivo da dor.

\section{MÉTODOS}

Foi realizada revisão sobre o tema na literatura em inglês e português em periódicos publicados no período de 2004 a 2014 na base de dados Medline. Foram utilizados na busca palavras-chave que identificassem condições de DCG: Transtornos somatoformes; Fibromialgia; Dor crônica; Dor musculoesquelética; Sistema hipófisesuprarrenal; Cortisol; ACTH; Hormônio Liberador da Corticotropina. Foram incluídos os trabalhos originais nos quais foi analisado o comportamento de um ou mais hormônios do EHHA em pacientes com DCG. Foram excluídos os artigos: 1) cujo sujeito da pesquisa não apresentava condição dolorosa crônica; 2) nos quais a condição dolorosa crônica estudada não era generalizada, mas localizada; 3) cuja população de estudo tinha uma doença específica (como hiperidrose primária, síndrome de Sjögren, distúrbios gastrointestinais funcionais etc.); 4) cuja população de estudo era restrita dentro do seu universo (exemplo: pacientes idosos com fibromialgia); 5) que não permitiram a correlação com o sintoma doloroso (como nos casos em que incluiu diversos sintomas somatoformes, não individualizando a forma dolorosa); 6) de revisão; 7) que não tinham como objetivo de estudo a avaliação do EHHA na condição dolorosa crônica (exemplo: artigo para avaliação de nova proposta estatística).

\section{RESULTADOS E DISCUSSÃO}

Após aplicação dos critérios de inclusão e exclusão, foram selecionados 22 artigos, todos de língua inglesa.

\section{Estado Cortisolêmico}

A pesquisa de artigos em apenas um único veículo de informação (MEDLINE) evidenciou a produção científica abundante nessa área. $O$ foco principal de estudo do EHHA na DCG é a condição de falta ou excesso de corticoide. A maneira utilizada pela maioria dos estudos foi a quantificação de cortisol livre salivar ou total sanguíneo (plasmático ou, menos frequente, sérico). É oportuno comentar que níveis de cortisol salivar correlacionam-se de forma confiável com concentrações séricas e plasmáticas de cortisol livre e podem ser obtidas com mínima interferência nas atividades diárias ${ }^{18,19}$. Um estudo, porém, se utilizou de quantificação de cortisol na urina de 24 horas $^{20}$.

Estudos mais antigos mostraram dados controversos ao evidenciar qual nível de cortisol era predominante nesses pacientes. A tabela 1 mostra os artigos dos últimos 10 anos que foram avaliados nesta revisão e que continuam não esclarecendo esse dado. Enquanto a maioria deles sugere hipocortisolismo ${ }^{20-23}$ e outros mostram uma tendência a hipocortisolismo ${ }^{24,25}$, outros não demonstram diferença ${ }^{26-}$ 29 e mais tantos ainda sugerem haver hipercortisolismo ${ }^{30-}$ 33. Essa questão é mais complexa do que parece, pois mesmo os artigos que mostram haver hipocortisolismo são contraditórios ao especificar o período. Por exemplo, enquanto McBeth et $\mathrm{al}^{22}$ mostraram haver nível de cortisol baixo de manhã e à noite, Riva et $\mathrm{al}^{21}$ conseguiram demonstrar esse mesmo resultado apenas ao acordar, mas não 30 e 60 minutos após o acordar e nem à noite. Ainda, Macedo et $\mathrm{al}^{25}$, Wingenfeld et $\mathrm{al}^{23}$ e Geiss et $\mathrm{al}^{29}$ testaram o nível de cortisol tanto na forma salivar quanto sanguínea; no entanto, enquanto o primeiro grupo mostrou hipocortisolismo na forma plasmática mas sem diferença na forma salivar, o segundo mostrou hipocortisolismo na forma salivar e sem diferença na forma plasmática; já o último mostrou não haver diferenças com ambas as formas.

A dificuldade em se determinar o nível de cortisol na DCG é ainda maior quando se observa como foram realizados os estudos. A Tabela 1 evidencia o número de indivíduos estudados, o tipo de cortisol quantificado e o nível de cortisol encontrado. É possível notar que o número de pacientes e controles é baixo na maioria deles. Além de haver divergência no tipo de cortisol quantificado, o mesmo se observa nos períodos de coleta. Alguns avaliam somente a manhã, enquanto outros analisam períodos ao longo do dia. As manhãs são determinadas pelo horário (6 horas, ou entre as 8 e 9 horas), mas também podem ser determinados pelo acordar (na hora do acordar, 30 ou 60 minutos após). Alguns estudos são rígidos, internando os indivíduos e determinando a hora de dormir, acordar e ter refeições; outros deixam o paciente realizar a autocoleta do cortisol salivar com $s w a b$, esperando que tenha sido feita na hora especificada. Por esses motivos, não é possível determinar o nível cortisolêmico na DCG.

\section{Outros Parâmetros do EHHA}

Outros parâmetros do EHHA também foram foco de investigação. No entanto, a atenção recebida foi menor. Um montante de resultados pode ser visualizado na tabela 2, que apresenta os estudos e os resultados quanto aos níveis de HLC, ACTH, teste de da supressão com ACTH, teste estressor e teste de sensibilidade ao corticoide. O teste de supressão com ACTH em baixa dose tem como objetivo avaliar o feedback negativo do 
eixo. No caso dos estudos apreciados nesta revista, foi feito o teste tanto em dose de $0,5 \mathrm{mg}$ quanto de $0,25 \mathrm{mg}$. $\mathrm{O}$ teste estressor foi utilizado para observar o aumento do cortisol frente a um evento estressor; foram utilizados pelos estudos testes físicos (que consistiam na pressão de determinados pontos até atingir o limiar doloroso) como psíquicos (um deles chegou a ser uma simulação de apresentação oral com avaliadores críticos). O teste de sensibilidade ao glicocorticoide foi feito de algumas maneiras diferentes, mas todas envolviam a produção de interleucina-6 (IL-6) induzida por lipopolissacarídeo (LPS) e quanto se conseguia inibir essa produção (o racional é que o corticoide tem ação imunossupressora e consequentemente reduz a produção de IL-6). Como se pode observar na tabela 2 , os achados são também bastante divergentes entre si, mas, diferentemente do nível de cortisol estudado, bem menos estudos avaliaram cada parâmetro.

TABELA 1 - Estado cortisolêmico na DCG e o respectivo autor principal e número de pacientes e controles do estudo

\begin{tabular}{|c|c|c|c|}
\hline Autor & $\mathbf{N}(\mathbf{P} \times \mathbf{C})$ & Tipo de Cortisol estudado & Achado \\
\hline Izquierdo-Alvarez ${ }^{20}$ & $47 \times 58$ & Urina $24 \mathrm{~h}$ & Hipocortisolismo \\
\hline Riva $^{21}$ & $29 \times 29$ & Livre salivar & $\begin{array}{l}\text { Hipocortisolismo (em momentos do dia, incluindo ao } \\
\text { acordar, mas não } 30 \text { e } 60 \mathrm{~min} \text { após, e não noturno) }\end{array}$ \\
\hline $\mathrm{McBeth}^{22}$ & $178 \times 80$ & Livre salivar & Hipocortisolismo (M e No) \\
\hline Wingenfeld ${ }^{23}$ & $18 \times 24$ & Livre salivar e total plasmático & Hipocortisolismo $(\mathrm{S})$ e sem diferença $(\mathrm{P})$ às $16 \mathrm{~h}$ \\
\hline Riva $^{24}$ & $29 \times 27$ & Livre salivar & Tendência a hipocortisolismo (M) \\
\hline Macedo $^{25}$ & $27 \times 29$ & Livre salivar e total plasmático & $\begin{array}{l}\text { Sem diferença (S) e tendência a hipocortisolismo (Pl) } \\
\text { (M) }\end{array}$ \\
\hline McLean $^{26}$ & $20 \times 16$ & Livre salivar & Sem diferença \\
\hline Wingenfeld ${ }^{27}$ & $15 \times 20$ & Plasmático & Sem diferença $(\mathrm{M})$ \\
\hline Aschbacher ${ }^{28}$ & $36 \times 36$ & Total plasmático & Sem diferença \\
\hline Geiss $^{29}$ & $12 \times 15$ & Livre salivar e total sérico & Sem diferença \\
\hline Fatima $^{30}$ & $40 \times 40$ & Total sérico & Hipercortisolismo (No) \\
\hline Bote $^{31}$ & $25 \times 20$ & Total plasmático & Hipercortisolismo (M) \\
\hline Wingenfeld $^{32}$ & $21 \times 26$ & Livre salivar & Hipercortisolismo (todo o dia) \\
\hline Bote $^{33}$ & $8 \times 8$ & Total plasmático & Hipercortisolismo (M) \\
\hline
\end{tabular}

TABELA 2 - Resultados quanto a demais parâmetros do EHHA avaliados nos estudos (referentes ao grupo estudado em relação aos controles)

\begin{tabular}{lllllll}
\hline Autor & N $(\mathbf{P} \times \mathbf{C})$ & $\begin{array}{l}{[\text { HLC] }} \\
\text { sérica }\end{array}$ & $\begin{array}{l}\text { [ACTH] } \\
\text { sérica }\end{array}$ & Teste de supressão ao ACTH & $\begin{array}{l}\text { Sensibilidade ao } \\
\text { CE }\end{array}$ & $\begin{array}{l}\text { Teste } \\
\text { estressor }\end{array}$ \\
\hline Bote $^{31}$ & $25 \times 20$ & $\downarrow$ & $\downarrow$ & - & - & - \\
McBeth $^{22}$ & $178 \times 80$ & - & - & Falha da inibição & SD \\
Wingenfeld $^{27}$ & $15 \times 20$ & - & SD & Aumento da inibição & - & - \\
Wingenfeld $^{23}$ & $18 \times 24$ & - & SD & $\downarrow$ & SD & $\downarrow$ \\
Aschbacher $^{28}$ & $36 \times 36$ & - & - & Falha da inibição & - & - \\
Geiss $^{29}$ & $12 \times 15$ & - & - & SD & $\downarrow$ & $\uparrow$ \\
Macedo $^{25}$ & $27 \times 29$ & - & - & - & SD & -
\end{tabular}

$\mathrm{N}=$ número de indivíduos estudados; $\mathrm{P}=$ pacientes; $\mathrm{C}=$ controles; HLC $=$ hormônio liberador da corticotrofina; ACTH $=$ hormônio adrenocorticotrófico; $\mathrm{CE}=$ corticoide; $\mathrm{SD}=$ sem diferença. 
De uma maneira original, Nees et al. ${ }^{34}$ optaram por avaliar o EHHA em pacientes com fibromialgia utilizando o condicionamento para a piscada de olho, que pode ser do tipo retardado (delay) ou de traço (trace). A piscada de olho condicionada funciona como um processo de aprendizado basal no qual o glicocorticóide tem capacidade de modular. Foram estudados 30 pacientes com fibromialgia e 20 controles saudáveis e foi observado que os primeiros apresentaram condicionamento da piscada de olho de traço facilitada e atraso no condicionamento retardado da piscada de olho. As medidas de cortisol correlacionaram-se significativamente com o condicionamento da piscada de olho de traço, com menores níveis de cortisol associados ao aumento desse tipo de condicionamento. Por outro lado, os níveis de cortisol não se correlacionaram com condicionamento retardado da piscada de olho.

\section{Dor versus Sintomas Psíquicos}

Apesar de se observar alguma disfunção do EHHA, com relação aos parâmetros acima descritos, na DCG, um questionamento foi habitualmente feito: tais alterações do eixo se relacionam com o sintoma doloroso ou com os sintomas psíquicos frequentemente apresentados por esses indivíduos? Alguns desses estudos tentaram responder a essa questão.

McLean et al. ${ }^{35}$ analisaram os níveis de HLC no líquor de 26 pacientes com fibromialgia, sem grupo controle. Níveis elevados do hormônio correlacionaramse com aumento dos sintomas dolorosos, mas não com fadiga.

Wingenfeld et al. ${ }^{32}$, porém, encontraram resultados contraditórios. Análises mostraram que as alterações do cortisol e do feedback do eixo apenas estavam presentes nas pacientes fibromiálgicas com depressão maior, não havendo relação com a redução do limiar doloroso vista nas pacientes com fibromialgia.

Um aspecto interessante foi estudado por McBeth et al. ${ }^{22}$. A equipe recrutou 2312 indivíduos com passagem em serviços de saúde primários e avaliou suas respostas sobre dor e sintomas psíquicos. Eles foram divididos em três grupos: um com DCG, um grupo "de risco", composto por indivíduos sem DCG, mas cujos sintomas psíquicos eram semelhantes aos do primeiro grupo, e um grupo controle, que não tinha evidência nem de DCG nem de estresse psicológico. Indivíduos selecionados aleatoriamente de cada grupo tiveram seu EHHA avaliado e foram novamente reavaliados 15 meses depois. Foi observado que os indivíduos "de risco" que, de fato, evoluíram para DCG apresentavam, inicialmente, redução do feedback inibitório do EHHA, baixos níveis de cortisol salivar pela manhã e altos níveis de cortisol salivar à noite, sendo fatores independentes e aditivos.

Os demais estudos que tentaram separar a correlação das alterações hormonais com os sintomas psíquicos ou com os sintomas dolorosos se mostraram contraditórios também nesse item ${ }^{26-29,32,35}$.

\section{Estudos dos Genes e Afins}

Além das avaliações acima descritas, Macedo et al. ${ }^{25}$ investigaram a expressão de ácido ribonucleico mensageiro (RNAm) dos receptores de corticoide (alfa e mineralocorticoide), da zipper leucina induzida por corticoide e da proteína ligadora FK506 5, além da investigação de polimorfismos dos receptores de corticoide. Foi observada uma menor expressão dos receptores de corticoide e de zipper leucina induzida por corticoide. Ainda, o alelo MR rs5522 (I180V) foi mais frequente em pacientes com fibromialgia e essa variação foi associada à perda de função do receptor.

Holliday et al. ${ }^{36}$ estudaram polimorfismos de nucleotídeo único (PNU) em sete genes de 994 indivíduos que estavam sendo seguidos prospectivamente, incluindo 164 com DCG e 172 sem dor. Múltiplos PNU no gene SERPINA6 foram associados ao número máximo de pontos dolorosos assim como a DCG. Outras associações significativas foram feitas com PNU nos genes CRHBP e POMC, assim como um PNU no gene MC2R. PNU também foram associados à baixa qualidade do sono e depressão, o que pode explicar algumas das associações observadas.

Por sua vez, Cizza et al. ${ }^{37}$ estudaram a prevalência de mutações do tipo nula/Lyon na vila italiana originária das primeiras pessoas com essa mutação. Esta ocorre no gene da proteína ligadora de cortisol no sangue, chamada globulina ligadora de corticosteroide (GLC). Mutações no gene da GLC, que são raras, podem levar a alterações nos níveis sanguíneos de cortisol. A pesquisa foi feita em 495 indivíduos, sendo detectada a mutação, na sua forma heterozigota, em 3,6\%. Destes, $44 \%$ tinham DCG e $17 \%$ tinham dor crônica.

\section{Intervenções na DCG e Comportamento do EHHA}

Alguns estudos realizaram intervenções na DCG e observaram o que mudava nos parâmetros do EHHA antes e após a intervenção. A tabela 3 lista os trabalhos, assim como descreve a intervenção realizada e as mudanças observadas no padrão de dor, do nível de cortisol e em outros parâmetros do EHHA após a intervenção. Apesar de haver poucos estudos, com tipos diferentes de intervenção e avaliação de diferentes parâmetros do EHHA, existe uma tendência de haver mudança do EHHA com a intervenção quando há mudança do padrão de dor, observado com intervenções mais duradouras em detrimento das pontuais. Este achado reforça uma associação da dor com o EHHA, ainda que isso não possa ser considerado uma associação direta. 
TABELA 3 - Lista evidenciando estudos que realizaram intervenção em indivíduos com DCG e os resultados da mesma na dor, no cortisol e outras ações

\begin{tabular}{llllll}
\hline Autor & N (P x C) & \multicolumn{1}{c}{ Intervenção } & Dor & Cortisol & \multicolumn{1}{c}{ Outras açães } \\
\hline Bonifazi $^{38}$ & $12 \times 0$ & AF/TCC/educação 3 sem & Melhora & Queda mais rápida & $\begin{array}{l}\uparrow \text { RNAm do } \\
\text { receptor CE }\end{array}$ \\
\hline Giske $^{39}$ & $19 \times 19$ & Contrações isométricas até exaustão & SD & SD & - \\
\hline Katedoff $^{40}$ & $16 \times 16$ & Contrações isométricas até exaustão & SD & SD & $\begin{array}{l}\uparrow \text { ACTH no } \\
\text { exercício }\end{array}$ \\
\hline Curtis $^{41}$ & $22 \times 0$ & Ioga 2x/sem por 8 sem & Melhora & $\begin{array}{l}\uparrow(\text { correlação com } \\
\text { dor) }\end{array}$ & - \\
\hline Bote $^{33}$ & $8 \times 8$ & 1 sessão ciclismo 45, & - & nas P e $\uparrow$ nos C & - \\
\hline
\end{tabular}

$\mathrm{N}=$ número de indivíduos estudados $; \mathrm{P}=$ pacientes $; \mathrm{C}=$ controles $; \mathrm{AF}=$ atividade física $; \mathrm{TCC}=$ terapia cognitivo-comportamental; sem $=$ semanas; $\mathrm{CE}=$ corticoide; $\mathrm{SD}=$ sem diferença .

\section{Considerações Gerais}

De uma maneira geral, encontramos vários estudos sobre a disfunção do EHHA na DCG. No entanto, a imensa maioria consiste de artigos com pequeno número de sujeitos, sugerindo haver uma fragilidade nos resultados que pode, inclusive, justificar a divergência nos resultados. Eles também apresentam diferenças em inúmeras variáveis e na forma como foram estudadas, o que dificulta a comparação entre eles. Pode-se fazer uma crítica também aos controles (quando existentes), também em número pequeno e nem sempre corretamente pareados.

\section{CONCLUSÃO}

Com todo o exposto acima, pode-se concluir que são fortes os indícios de existência de alterações hormonais do EHHA nos indivíduos com DCG. Esses achados, porém, são divergentes, não sendo claro qual é a real disfunção do eixo nesses pacientes. Não se pode afirmar se essas alterações observadas estão de fato associadas à condição dolorosa ou se aos sintomas psíquicos frequentemente associados a essas condições. Da mesma forma, não é possível caracterizar se as alterações do EHHA são a causa ou reflexo da DCG. Estudos genéticos sugerem que elas podem ser, sim, a origem desses distúrbios. Mais estudos são necessários e com maior número de sujeitos e melhor pareamento. Por ora, portanto, não é possível para o médico perito utilizar os parâmetros objetivos da disfunção do EHHA como método auxiliar na avaliação da incapacidade laborativa de indivíduos com DCG.

Nascimento IS; Miziara CSMG. Hypothalamus-Pituitary-Adrenal axis dysfunction in chronic widespread pain: literature analysis and disability evaluation. Saúde, Ética \& Justiça. 2015;20(1):29-36.

ABSTRACT: Pain is a common disturbance and a frequent cause of work sick leave. Because the symptom is subjective (perceived), it hinders the expert evaluation. Chronic musculoskeletal pain, in particular, can lead to prolonged absenteeism, which places a substantial economic burden on employers and society. Therefore, forensic physicians, who evaluate work incapacity, take upon themselves a critically important task. A greater comprehension of the mechanisms that generate these clinical conditions, which include fibromyalgia and somatoform pain disorder, can help turn these mostly subjective assessments in more objective ones and consequently facilitate work capacity appraisal. Accordingly, scientific literature on hypothalamus-pituitary-adrenal axis function in the context of chronic generalized musculoskeletal pain published in the last 10 years was reviewed, but earlier articles were included for their relevance. It was noted that hormonal disturbances of this axis were not rare, but results are contradictory and it is not clear if the association occurs with pain or with the psychological symptoms frequently associated with these disorders. More studies are needed with a larger subject group.

KEY WORDS: Somatoform Disorders; Fibromyalgia; Chronic Pain; Musculoskeletal Pain; Pituitary-Adrenal System; Hydrocortisone, Adrenocorticotropic Hormone; Corticotropin-Releasing Hormone.

\section{REFERÊNCIAS}

1. Wolfe F, WalittBT, KatzRS, Häuser W. Social Security Work Disability and its Predictors in Patients with Fibromyalgia.
Arthritis Care Res (Hoboken). 2014; 66(9):1354-63. DOI: http://dx.doi.org/10.1002/acr.22305 
2. American Society of Anesthesiologists Task Force on Chronic Pain Management, American Society of Regional Anesthesia and Pain Medicine. Practice Guidelines for Chronic Pain Management: an updated report by the American Society of Anesthesiologists Task Force on Chronic Pain Management and the American Society of Regional Anesthesia and Pain Medicine. Anesthesiology. 2010; 112(4):810-33. DOI: http://dx.doi.org/ 10.1097/ ALN.0b013e3181c43103

3. American Psychiatric Association - APA. Diagnostic and Statistical Manual of Mental Disorders - DSM-5. 5th ed. Arlington, VA: American Psychiatric Association; 2013.

4. American Psychiatric Association - APA. Diagnostic and Statistical Manual of Mental Disorders - DSM-4. 4th ed. Arlington, VA: American Psychiatric Association; 2002.

5. Wolfe F, Clauw DJ, Fitzcharles MA, Goldenberg DL, Katz RS, Mease P, et al. The American College of Rheumatology Preliminary Diagnostic Criteria for Fibromyalgia and Measurement of Symptom Severity. Arthritis Care Res (Hoboken). 2010; 62(5):600-10. DOI: http://dx.doi. org/10.1002/acr.20140

6. Wolfe F, Smythe HA, Yunus MB, Bennett RM, Bombardier C, Goldenberg DL, et al. The American College of Rheumatology 1990 criteria for the classification of fibromyalgia: a report of the Multicenter Criteria Committee. Arthritis Rheum. 1990; 33(2):160-72.

7. Häuser W, Henningsen P. Fibromyalgia syndrome: a somatoform disorder? Eur J Pain.2014; 18(8):1052-9. DOI: http://dx.doi.org/10.1002/j.1532-2149.2014.00453.x

8. Egloff N, Cámara RJA, von Känel R, Klingler N, Marti E, Ferrari ML. Hypersensitivity and hyperalgesia in somatoform pain disorders. Gen Hosp Psychiatry. 2014; 36(3):284-90. DOI: http://dx.doi.org/10.1016/j. genhosppsych.2014.01.011

9. Wolfe F, Walitt BT, Katz RS, Häuser W. Symptoms, the Nature of Fibromyalgia, and Diagnostic and Statistical Manual 5 (DSM-5) Defined Mental Illness in Patients with Rheumatoid Arthritis and Fibromyalgia. PLoS One. 2014; 9(2):e88740. DOI: http://dx.doi.org/10.1371/journal. pone. 0088740

10. Collado A, Gomez E, Coscolla R, Sunyol R, Solé E, Rivera $\mathrm{J}$, et al. Work, family and social environment in patients with Fibromyalgia in Spain: an epidemiological study: EPIFFAC study. BMC Health Serv Res. 2014; 14:513. DOI: http://dx.doi.org/10.1186/s12913-014-0513-5

11. Linares MCU, Pérez IR, Pérez MJB, Lima AOL, Castaño JP. Características clínicas y psicosociales de personas con fibromialgia. Repercusión del diagnóstico sobre sus actividades. Rev Esp Salud Pública. 2005; 79(6):683-95.

12. Apkarian AV, Baliki MN, Geha PY. Towards a theory of chronic pain. Prog Neurobiol. 2009; 87(2):81-97. DOI: http://dx.doi.org/10.1016/j.pneurobio.2008.09.018

13. Smith SM, Vale WW. The role of the hypothalamicpituitary-adrenal axis in neuroendocrine responses to stress. Dialogues Clin Neurosci. 2006; 8(4):383-95.

14. Chrousos GP. The hypothalamic-pituitary-adrenal axis and immune-mediated inflammation. N Engl J Med. 1995; 332(20):1351-62.

15. Guyton AC, Hall JE. Tratado de Fisiologia Médica. $10^{\mathrm{a}}$ ed. Rio de Janeiro: Guanabara Koogan; 2002. p.818-22.
16. Crofford LJ. The hypothalamic-pituitary-adrenal stress axis in fibromyalgia and chronic fatigue syndrome. $\mathrm{Z}$ Rheumatol. 1998; 57(Suppl.2):67-71.

17. Lariviere WR, Melzack R. The role of corticotropinreleasing factor in pain and analgesia. Pain. 2000; 84(1):112.

18. Kirschbaum C, Hellhammer DH. Salivary cortisol in psychoneuroendocrine research: recente developments and applications. Psychoneuroendocrinology. 1994; 19(4):31333.

19. Kirschbaum C, Hellhammer DH. Salivary cortisol in psychobiological research: an overview. Neuropsychobiology. 1989; 22(3):150-69.

20. Izquierdo-Alvarez S, Bocós-Terraz JP, Bancalero-Flores JL, Pavón-Romero L, Serrano-Ostariz E, de Miguel CA. Is there an association between fibromyalgia and belownormal levels of urinary cortisol? BMC Res Notes. 2008; 1:134. DOI: http://dx.doi.org/10.1186/1756-0500-1-134

21. Riva R, Mork PJ, Westgaard RH, Rø M, Lundberg U. Fibromyalgia syndrome is associated with hypocortisolism. Int J Behav Med. 2010; 17(3):223-33. DOI: http://dx.doi. org/10.1007/s12529-010-9097-6

22. McBeth J, Silman AJ, Gupta A, Chiu YH, Ray D, Morriss $\mathrm{R}$, et al. Moderation of psychosocial risk factors through dysfunction of the hypothalamic-pituitary-adrenal stress axis in the onset of chornic widespread musculoskeletal pain: findings of a population-based prospective cohort study. Arthritis Rheum. 2007; 56(1):360-71.

23. Wingenfeld K, Heim C, Schmidt I, Wagner D, Meinischmidt G, Hellhammer DH. HPA axis reactivity and lymphocyte glucocorticoid sensitivity in fibromyalgia syndrome and chronic pelvic pain. Psychosom Med. 2008; 70(1):65-72.

24. Riva R, Mork PJ, Westgaard RH, Lundberg U. Comparison of the cortisol awakening response in women with shoulder and neck pain and women with fibromyalgia. Psychoneuroendocrinology 2012; 37(2):299-306. DOI: http://dx.doi.org/10.1016/j.psyneuen.2011.06.014

25. Macedo JA, Hesse J, Turner JD, Meyer J, Hellhammer $\mathrm{DH}$, Muller CP. Glucocorticoid sensitivity in fibromyalgia patients: decreased expression of corticosteroid receptors and glucocorticoid-induced leucine zipper. Psychoneuroendocrinology. 2008; 33(6):799-809. DOI: http://dx.doi.org/10.1016/j.psyneuen.2008.03.012

26. McLean SA, Williams DA, Harris RE, Kop WJ, Groner KH, Ambrose K, et al. Momentary relationship between cortisol secretion and symptoms in patients with fibromyalgia. Arthritis Rheum. 2005; 52(11):3660-9.

27. Wingenfeld K, Wagner D, Schmidt I, Meinischmidt G, Hellhammer DH, Heim C. The low-dose dexamethasone suppression test in fibromyalgia. J Psychosom Res. 2007; 62(1):85-91.

28. Aschbacher K, Adam EK, Crofford LJ, Kemeny ME, Demitrack MA, Ban-Zvi A. Linking disease symptoms and subtypes with personalized systems-based phenotypes: a proof of concept study. Brain Behav Immun. 2012; 26(7):1047-56. DOI: http://dx.doi.org/10.1016/j. bbi.2012.06.002

29. Geiss A, Rohleder N, Anton F. Evidence for an association between an enhanced reactivity of interleukin- 6 levels and reduced glucocorticoid sensitivity in patients with 
fibromyalgia. Psychoneuroendocrinology. 2012; 37(5)67184. DOI: http://dx.doi.org/10.1016/j.psyneuen.2011.07.021

30. Fatima G, Das SK, Mahdi AA, Verma NS, Khan FH, Tiwari AM, et al. Circadian rhythm of serum cortisol in female patients with fibromyalgia syndrome. Indian J Clin Biochem. 2013; 28(2):181-4. DOI: http://dx.doi.org/10.1007/s12291012-0258-z

31. Bote ME, García JJ, Hinchado MD, Ortega E. Inflammatory/ stress feedback dysregulation in women with fibromyalgia. Neuroimmunomodulation. 2012; 19(6):343-51. DOI: http:// dx.doi.org/10.1159/000341664

32. Wingenfeld K, Nutzinger D, Kauth J, Hellhammer DH, Lautenbacher S. Salivary cortisol release and hypothalamic pituitary adrenal axis feedback sensitivity in fibromyalgia is associated with depression but not with pain. J Pain. 2010; 11(11):1195-202. DOI: http://dx.doi.org/10.1016/j. jpain.2010.02.011

33. Bote ME, Garcia JJ, Hinchado MD, Ortega E. Fibromyalgia: antiinflammatory and stress responses after acute moderate exercise. PLoS One. 2013; 8(9):e74524. DOI: http://dx.doi. org/10.1371/journal.pone.0074524

34. Nees F. Ruddel H, Mussgay L, Kuehl LK, Römer S, Schächinger H. Alteration of delay and trace eyeblink conditioning in fibromyalgia patients. Psychosom Med. 2010; 72(4):412-8. DOI: http://dx.doi.org/10.1097/ PSY.0b013e3181d2bbef

35. McLean SA, Williams DA, Stein PK, Harris RE, Lyden AK, Whalen G, et al. Cerebrospinal fluid corticotropinreleasing factor concentration is associated with pain but not fatigue symptoms in patients with fibromyalgia. Neuropsychopharmacology. 2006; 31(12):2776-82.

36. Holliday KL, Nicholl BI, Macfarlane GJ, Thomson W,
McBeth J. Genetic variation in the hypothalamic-pituitaryadrenal stress axis influences susceptibility to usculoskeletal pain: results from the EPIFUND study. Ann Rheum Dis. 2010; 69(3):556-60. DOI: http://dx.doi.org/10.1136/ ard.2009.116137

37. Cizza G, Bernardi L, Smirne N, Maletta R, Tomaino C, Costanzo A, et al. Clinical manifestations of highly prevalent corticosteroid-binding globulin mutations in a village in souther Italy. J Clin Endocrinol Metab. 2011; 96(10):E1684-93. DOI: http://dx.doi.org/10.1210/jc.20111321

38. Bonifazi M, Suman AL, Cambiaggi C, Felici A, Grasso G, Lodi L, et al. Changes in salivary cortisol and corticosteroid receptor-alpha mRNA expression following a 3-week multidisciplinary treatment program in patients with fibromyalgia. Psychoneuroendocrinology. 2006; 31(9):1076-86.

39. Giske L, Vøllestad NK, Mengshoel AM, Jensen J, Knardahl S, Røe C. Attenuated adrenergic responses to exercise in women with fibromyalgia - a controlled study. Eur J Pain. 2008; 12(3):351-60.

40. KadetoffD, Kosek E. Evidence of reduced sympatho-adrenal and hypothalamic-pituitary activity during static muscular work in patients with fibromyalgia. J Rehabil Med. 2010; 42(8):765-72. DOI: http://dx.doi.org/10.2340/165019770597

41. Curtis K, Osadchuk A, Katz J. An eight-week yoga intervention is associated with improvements in pain, psychological functioning and mindfulness, and changes in cortisol levels in women with fibromyalgia. J Pain Res 2011; 4:189-201. DOI: http://dx.doi.org/10.2147/JPR. S22761 